\title{
A THEOREM ON MONOTONE INTERIOR TRANSFORMATIONS
}

\author{
EDWIN E. MOISE
}

B. Knaster ${ }^{1}$ has raised the question whether there is a compact metric continuum $M$, irreducible between two of its points, and a monotone interior transformation $T$, throwing $M$ into the unit interval, such that for each $x$ of $T(M), T^{-1}(x)$ is an arc. In the present note, we shall answer this question in the negative.

Suppose that such a continuum exists, and let $T(M)=I=[0,1]$. Let $K$ be a subcontinuum of $M$ which contains points of $T^{-1}(x)$ and $T^{-1}(y)$, where $x, y \in I$ and $x \neq y$. Since $M$ is an irreducible continuum, $K$ contains $T^{-1}(z)$ for each $z$ between $x$ and $y$; and since $T$ is interior, $K$ contains $T^{-1}(x)$ and $T^{-1}(y)$. It follows that each subcontinuum of $M$ either is an arc or contains an open subset of $M$, but not both. In the first case, $K$ lies in the inverse image of a point of $I$, and in the second case, $K$ is the inverse image of a subinterval of $I$. In either case, $K$ is decomposable.

Now let $C_{1}$ be a simple chain of open subsets of $M$, with links $C_{1}$, $c_{2}, \cdots, c_{k}$, covering $T^{-1}(0)$, such that each link of $C_{1}$ contains a point of $T^{-1}(0)$ which does not lie in the closure of the sum of the other links of $C_{1}$. There is a subcontinuum $K_{1}$ of $M$, lying in $\sum c_{i}$ and containing $T^{-1}(0)$, such that for each link $c$ of $C_{1}$, each component of $K_{1}-c \cdot K_{1}$ is a boundary subset of $M$; each such component is therefore an arc. Let $K$ be $T^{-1}\left(I^{\prime}\right), I^{\prime} \subset I$. For each $x$ of $I^{\prime}, \bar{c}_{k}$ is the sum of two mutually exclusive closed point-sets $H$ and $H^{\prime}$, containing $\bar{c}_{k} T^{-1}(0)$ and $\bar{c}_{k} T^{-1}(x)$ respectively. In fact, for each $j<k$, the closure of $c_{2}+c_{3}+\cdots+c_{j}$ has a separation into closed sets which induces such a separation of $\bar{c}_{k}$. But the closure of ${ }^{2} C_{1}^{*}$ obviously has no such separation; whence it follows that there is a component $L$ of $K_{1}-K_{1} \cdot \bar{c}_{k}$ which has a limit-point in $H$ and a limit-point in $H^{\prime} . L$ must contain a point not in the closure of $c_{2}+c_{3}+\cdots+c_{k-1}$; and being a boundary set, $L$ is an arc. $L$ is therefore a subset of the inverse image of a point $y$ of $I$. Let $A_{1}$ be $T^{-1}(0)$, and let $A_{2}$ be $T^{-1}(y)$.

By repeated application of the above procedure, we obtain a sequence $A_{1}, A_{2}, \cdots$ of arcs lying in $M$, and a sequence $C_{1}, C_{2}, \cdots$ of simple chains of open subsets of $M$, such that (1) $C_{i}$ covers $A_{i}$, (2)

Presented to the Society, April 17, 1948; received by the editors June 10, 1948.

${ }^{1} \mathrm{~B}$. Knaster, Un continu irréductible à décomposition continue en tranches, Fund. Math. vol. 25 (1935) p. 577.

${ }^{2}$ If $C$ is a collection of sets, then $C^{*}$ denotes the sum of the elements of $C$. 
the closure of $C_{i+1}^{*}$ lies in $C_{i}^{*}$, (3) the maximum diameter of the links of $C_{i}$ is less than $1 / i$, and (4) $C_{i+1}$ contains two mutually exclusive chains, each of which has a link in each link of $C_{i}$.

Let $N$ be the common part of the closures of the sets $C_{i}^{*}$. Suppose that $N$ is the sum of two mutually exclusive closed point-sets $H$ and $H^{\prime}$. Condition (3) now implies that for some $i, C_{i}$ is the sum of two mutually exclusive open sets each of which is a sum of links of $C_{i}$; since $C_{\boldsymbol{i}}$ is a simple chain, this is impossible. Therefore $N$ is a continuum.

It is not difficult to show that $N$ is indecomposable. The proof indicated below is very similar to a proof given in another connection by Knaster. ${ }^{3}$ We wish to show that if $N^{\prime}$ is a proper subcontinuum of $N$, then every point of $N^{\prime}$ is a limit-point of $N-N^{\prime}$. For each $i$, let $C_{\boldsymbol{i}}^{\prime}$ be the set of all links of $C_{i}$ that contain a point of $N^{\prime}$. There is a $k$ such that for $i>k, C_{i}-C_{i}^{\prime}$ contains two intersecting links of $C_{i}$. It follows that for $i>k, C_{i+2}$ contains two mutually exclusive chains, one of which covers $N^{\prime}$ and both of which have a link in each link of $C_{i+1}$. Therefore $N$ is the closure of $N-N^{\prime}$. Since $M$, by hypothesis, contains no indecomposable continuum, the proof of the following theorem is now complete:

THEOREM. For no compact, metric, irreducible continuum $M$ is there a monotone interior transformation throwing $M$ into an arc $A$ such that the inverse image of each point of $A$ is an arc.

UNIVERSITY OF MICHIGAN

${ }^{3} \mathrm{~B}$. Knaster, Un continu dont tout sous-continu est indécomposable, Fund. Math. vol. 3 (1922) p. 279. 